\title{
The Volumetric Singular Value and Robustness of Feedback Control Systems
}

\author{
B. R. Barmish* and B. T. Polyak** \\ *ECE Department \\ University of Wisconsin-Madison \\ Madison, WI 53706 \\ ** Moscow Institute of Control Sciences \\ Moscow, Russia
}

\begin{abstract}
This short paper provides an overview of results which are fully described in [7]. The focal point is a new concept - the volumetric singular value $\mu_{v}$. In contrast to the theory underlying the structured singular value $\mu$, the volumetric theory includes no implicit assumption that all components $\Delta_{i}$ of the uncertainty $\Delta$ are expanded by the same factor $r \geq 0$. In making the transition from stability to instability, we allow for separate bounds $r_{i}$ for each component $\Delta_{i}$ of $\Delta$. Within this new framework, it becomes possible to systematically study the tradeoffs associated with various uncertainty components. To this end, given a complex $n \times n$ matrix $M$ and a positive diagonal matrix $R$ of uncertainty bounds, we provide a definition of $\mu_{v}(M)$ which involves maximization of the natural volumetric measure $\operatorname{vol}(R) \doteq(\operatorname{det} R)^{1 / n}$ subject to the usual stability preservation constraint $\operatorname{det}(I+M \Delta \neq 0)$. From a computational point of view, it turns out that $\mu_{v}(M)$ enjoys many of the nice properties enjoyed by $\mu(M)$.

\section{Summary}

In this short paper, we provide a summary of a number of technical results which are fully described in [7]. The motivation for this research is quite simple: Traditional robustness measures such as the multivariable stability margin (see [1] and [2]) are not informative about what tradeoffs are available among various components of the uncertainty. That is, the robustness measure is obtained by assuming that the same expansion factor $r$ is applied to all components $\Delta_{i}$ of the uncertainty $\Delta$. More specifically, in a traditional robustness margin computation, the objective is to calculate
\end{abstract}

$r_{\max } \doteq \sup \{r$ : robust stability is guaranteed for $\|\Delta\| \leq r\}$

where $\|\Delta\|$ is some suitably defined norm on the appropriate product space for the uncertainty $\Delta=$ $\left(\Delta_{1}, \Delta_{2}, \ldots, \Delta_{\ell}\right)$. Equivalently, one can study

$$
\mu \doteq \frac{1}{r_{\max }}
$$

when working in the structured singular value (SSV) framework.

With regard to uncertainty tradeoffs, the key point to note is that in SSV analysis, each component $\Delta_{i}$ of $\Delta$ above is scaled by the identical factor $r$. Said another way, the shape of the uncertainty set is assumed to be fixed and the robustness margin is obtained by magnifying the uncertainty by the same factor in all directions. Roughly speaking, if one views the uncertainty as a box, a classical $\mu$ calculation involves fixing the aspect ratios of this box apriori. In many cases, this leads to a value of $r_{\max }$ which may dramatically understate the degree to which the system is robust.

Researchers have long recognized the fact that working with separate uncertainty bounds $r_{i}$ for each component $\Delta_{i}$ adds extra degrees of freedom which can be exploited to provide more information about the robustness of a system; such an observation goes at least as far back as [3]. For example, in a typical robustness analysis, one might pose the following question: For a $20 \%$ decrease in the uncertainty bound for $\Delta_{1}$, what increases can be tolerated in the uncertainty bounds for $\Delta_{2}, \Delta_{3}, \ldots, \Delta_{\ell}$ while preserving stability? To answer this question via traditional robustness analysis meth ods, some sort of iterative scheme (perhaps involving a degree of trial and error) is needed because the shape of uncertainty bounding set is fixed and calculations are performed with respect to just one parameter the radial expansion factor $r$. In two recent papers (see [4] and [5]), a second parameter $\beta$ is introduced to parameterize the uncertainty bounding set. The Schur stability problem is considered and the objective is to optimize both $r$ and $\beta$ to enhance robustness.

In contrast to existing work, the main objective of this new line of research is to provide a framework for systematic study of the type of tradeoffs described above - a framework which allows an arbitary number of degrees of freedom for shaping the uncertainty bounding set. The takeoff point for our analysis is a new concept - the volumetric singular value $\mu_{v}$ which we often refer to as the VSV. Like the SSV, we work 
with the classical $(M, \Delta)$ configuration as in Doyle [1]. However, in contrast to the SSV which involves just one parameter $r$ to describe expansion of the uncertainty, the VSV theory involves an uncertainty bounding set described by $\ell$ parameters $r_{1}, r_{2}, \ldots, r_{\ell}$.

More specifically, we consider an $n \times n$ diagonal matrix of positive uncertainty bounds

$$
R=\operatorname{diag}\left\{r_{1}, \ldots, r_{2}, \ldots, r_{\ell}, \ldots\right\}
$$

where each $r_{i}$ is repeated $n_{i}$ times and $n_{i}$ is the dimension of $\Delta_{i}$. In other words, $n=n_{1}+\cdots+n_{\ell}$. For a given $n \times n$ complex matrix $M$, we obtain the VSV by maximizing the natural volumetric measure

$$
\operatorname{vol}(R) \doteq\left(\prod_{i=1}^{\ell} r_{i}^{n_{i}}\right)^{\frac{1}{n}}=(\operatorname{det} R)^{\frac{1}{n}}
$$

subject to the usual stability preservation constraint

$$
\operatorname{det}(I+M \Delta) \neq 0 \text {. }
$$

Subsequently, we take

$$
\mu_{v}(M)=\frac{1}{v o l_{\max }(M)}
$$

where $v_{\text {max }}(M)$ denotes the maximal achievable volume above.

From a technical point of view, it turns out to be convenient to describe the stability constraint in the space of bounds $\left(r_{1}, r_{2}, \ldots, r_{\ell}\right)$ rather than in the product space associated with the uncertainty $\Delta$. Perhaps the best example illustrating the power of bound space analysis is the rank one complex case for $M$. Although the set of $\Delta$ rendering $\operatorname{det}(I+M \Delta)$ nonsingular can be highly nonconvex, we prove that in bound space, the set of $\left(r_{1}, r_{2}, \ldots, r_{\ell}\right)$ guaranteeing closed loop stability is convex. This fact is exploited in the computation of $\mu_{v}(M)$.

The full version of this paper (see [7]) is summarized as follows: In Section 2, we provide a simple $2 \times 2$ example to illustrate the "power" of the VSV formulation vis-a-vis the SSV. This leads quite naturally to the formalism of Section 3 - the more general $n \times n$ framework is described and some lemmas are given relating the VSV and the SSV. For example, to make a quantitative comparison between the VSV and the SSV, we note that $\mu(M)$ can be associated with a suboptimal solution to the volume problem. Namely, if we think of the shape of the uncertainty bounding set as being already absorbed into the matrix $M$, then we can think of an SSV calculation as a suboptimal VSV calculation with the added constraint

$$
r_{1}=r_{2}=\cdots=r_{\ell} \text {. }
$$

Hence, with $r_{\max }(M)=1 / \mu(M)$, it is easy to see that the inequality

$$
\operatorname{vol}_{\max }(M)=\frac{1}{\mu_{v}(M)} \geq \frac{1}{\mu(M)}=r_{\max }(M)
$$

is quite meaningful.

Sections 4 and 5 are aimed at making the case that $\mu_{v}(M)$ enjoys pretty much the same convexity and computability properties as $\mu(M)$. For example, in the rank one case involving affine linear uncertainty structures, we provide precise formulae for $\mu_{v}(M)$ and, in the more general case, we arrive at results which are readily comparable to those in $\mu$ theory; i.e., we obtain a convex upper bound which involves both a scaling matrix $D$ as in SSV theory and the uncertainty bounding matrix $R$ which is particular to VSV theory. This is accomplished by refining the proof of Sezginer and Overton [6] to establish an upper bound for $\mu_{v}(M)$ which is jointly convex in the pair $(R, D)$.

In Section 6 , the focal point is taking the infimum of the VSV with respect to frequency an an important subtlety associated with the determination of a volume maximizing matrix. Finally, conclusions are drawn in Section 7. Of particular interest is the possibility of working with VSV in a synthesis context.

Acknowledgement

This research supported by the National Science Foundation under Grant No. ECS-9111570.

\section{References}

[1] J. C. Doyle, "Analysis of Feedback Systems with Structured Uncertainties," IEE Proceedings, vol. 129, Part D, no. 6, pp.242-250, 1982.

[2] M. G. Safonov, "Stability Margins of Diagonally Perturbed Multivariable Feedback Systems," IEE Proceedings, vol. 129, Part D, no. 6, pp. 251-256.

[3] I. Horowitz, "Quantitative Feedback Theory," IEE Proceedings, vol. 129, Part D, no. 6, pp.215-226, 1982.

[4] Y. Q. Shi, K. K. Yen and D. F. Zhang, "Improved Stability Robustness of Linear Discrete-Time Systems Via a Linear Fractional Transformation," IEEE Transactions on Industrial Electronics, vol. 37, pp. 538-543, 1990 .

[5] Q. H. Wu and M. Mansour, "On the Stability Hyperellipsoid of a Schur Polynomial," to appear.

[6] R. Sezginer and M. Overton, "The Largest Singular Value of $e^{x} A e^{-x}$ is Convex on Convex Sets of Commuting Matrices," IEEE Transactions on Automatic Control, vol. AC-35, no. 2, pp. 229-230, 1990.

[7] B. R. Barmish and B. T. Polyak, "The Volumetric Singular Value and Robustness of Feedback Control Systems," Technical Report ECE-93-9, ECE Department, University of Wisconsin-Madison, September 1993 . 